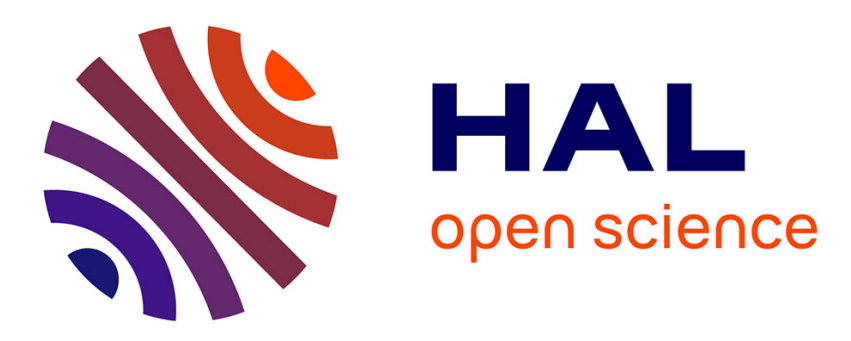

\title{
Differentiation Between Short and Long TCP Flows: Predictability of the Response Time
}

Konstantin Avrachenkov, Urtzi Ayesta, Patrick Brown, Eeva Nyberg

\section{To cite this version:}

Konstantin Avrachenkov, Urtzi Ayesta, Patrick Brown, Eeva Nyberg. Differentiation Between Short and Long TCP Flows: Predictability of the Response Time. [Research Report] RR-4964, INRIA. 2003. inria-00071615

\section{HAL Id: inria-00071615 https://hal.inria.fr/inria-00071615}

Submitted on 23 May 2006

HAL is a multi-disciplinary open access archive for the deposit and dissemination of scientific research documents, whether they are published or not. The documents may come from teaching and research institutions in France or abroad, or from public or private research centers.
L'archive ouverte pluridisciplinaire HAL, est destinée au dépôt et à la diffusion de documents scientifiques de niveau recherche, publiés ou non, émanant des établissements d'enseignement et de recherche français ou étrangers, des laboratoires publics ou privés. 
INSTITUT NATIONAL DE RECHERCHE EN INFORMATIQUE ET EN AUTOMATIQUE

\title{
Differentiation Between Short and Long TCP Flows: Predictability of the Response Time
}

\author{
Konstantin Avrachenkov — Urtzi Ayesta — Patrick Brown — Eeva Nyberg
}

\section{$\mathbf{N}^{\circ} 4964$}

Octobre 2003

THÈME 1 



\title{
Differentiation Between Short and Long TCP Flows: Predictability of the Response Time
}

\author{
Konstantin Avrachenkov*, Urtzi Ayesta ${ }^{\dagger}$, Patrick Brown, Eeva Nyberg ${ }^{\ddagger}$ \\ Thème 1 - Réseaux et systèmes \\ Projets MISTRAL
}

Rapport de recherche $\mathrm{n}^{\circ} 4964$ - Octobre 2003 - 26 pages

\begin{abstract}
:
Internet measurements show that a small number of large TCP flows are responsible for the largest amount of data transferred, whereas most of the TCP sessions are made up of few packets. Several authors have invoked this property to suggest the use of scheduling algorithms which favor short jobs, such as $L A S$ (Least Attained Service), to differentiate between short and long TCP flows.

We propose a packet level stateless, threshold based scheduling mechanism for TCP flows, RuN2C. We describe an implementation of this mechanism which has the advantage of being TCP compatible and progressively deployable. We compare the behavior of RuN2C with $L A S$ based mechanisms through analytical models and simulations of TCP in networks with tail drop buffers. In addition to studying the mean response time conditioned on given flow size, the principle performance measure used in previous studies, we also study the impact of the scheduling mechanisms on extreme values of response times as well as on the stability of the network, illustrated by the mean number of connections in the system.

We show that RuN2C has a very beneficial effect on the delay of short flows, while treating large flows as the current TCP scheduling does. In contrast, we find that $L A S$ based mechanisms can lead to pathological behavior in extreme cases.
\end{abstract}

Key-words: $\quad \mathrm{TCP} / \mathrm{IP}, M / G / 1$, Processor Sharing, $L A S$, Response time, Simulations, Queueing Theory

* INRIA, 2004 route des Lucioles, B.P.93, 06902, Sophia Antipolis Cedex, France. E-mail: k.avrachenkov@sophia.inria.fr

$\dagger$ France Telecom R\&D, 905 rue A. Einstein, 06921 Sophia Antipolis Cedex France. E-mail: $\{$ Patrick.Brown,Urtzi.Ayesta\}@francetelecom.com

$\ddagger$ Networking Laboratory, Helsinki University of Technology, Finland. E-mail: eeva.nyberg@hut.fi 


\section{Différentiation entre des connexions TCP de durées courtes et longues: conséquences sur les temps de réponse.}

\section{Résumé :}

Les observations de trafic sur l'Internet montrent que les connexions de longues durées génèrent une grande proportion du trafic TCP. Cependant, ces mêmes études montrent aussi que la majorité des flux TCP ont une durée de transmission très courte. Hors ces connexions courtes sont très vulnérables aux pertes de paquets.

Dans ce rapport, nous présentons un nouveau mécanisme d'ordonnancement de flux TCP qui donne une priorité plus importante aux premiers paquets d'une connexion, favorisant ainsi les connexions courtes. Ce mécanisme a la particularité de ne pas nécessiter le maintien en mémoire d'un état par flux. Nous montrons, à travers des analyses et des simulations, que le délai de transfert résultant pour les connexions de courte durée est réduit sans pour autant pénaliser les performances des autres connexions.

Mots-clés : $\mathrm{TCP} / \mathrm{IP}, M / G / 1$, Processor Sharing, LAS, Temps du réponse, Simulation NS, théorie des la files d'attente. 


\section{Introduction}

We study the differentiation between short and long flows in a TCP/IP network. In contrast to previous works, we show through analytical results and simulation that a threshold based mechanism is not only a convenient and implementable alternative to the Least Attained Service $(L A S)$ or Foreground-Background $(F B)$ scheduling policies, but that it also avoids several drawbacks presented by implementations of such mechanisms.

There are several reasons to favor short flows, arguments not completely uncorrelated. The arguments are based on user ergonomics, on the design of TCP congestion control, on the file size distribution and on queueing theory. Most user interactions with a network or with applications running across a network consist either entirely of short interchanges or, of short interchanges followed by a longer transfer. If the sum of transfer times were constant, from an ergonomics point of view, it would seem preferable to diminish the transfer times of short transactions at the expense of transfer times of longer transactions, as they do not require as much user attention as short transactions.

TCP applications react to congestion and losses by reducing their window sizes either with a certain fluidity through fast retransmit procedures or after a timeout. For short connections with small window sizes, a loss is often detected only after a timeout and possibly after all data has been sent to the network. As a result, timeouts on short connections are not very effective in reducing the overall traffic and stabilizing the network. Even less so, as short connections have been shown to represent a small percentage of overall traffic. A loss occurrence for a short TCP transfer may thus increase the transfer time manifold while, on the other hand, a reduction in the latency of the order of one second would be a significant improvement [1].

From a queueing theory point of view, it has been shown that choosing an appropriate scheduling policy may significantly improve the performance of the system. One of the classical results of queuing theory says that the Shortest Remaining Processing Time (SRPT) policy is optimal for the network as it is able to reduce the overall mean latency of the flows [2]. This is only applicable, if there is use of a priori knowledge of the flow sizes, which obviously is not the case in the current TCP/IP architecture. When the size jobs is not known, $L A S$ has been proposed as a good aproximation of SRPT. Yashkov showed [3] that $L A S$ scheduling policy is optimal with respect to the average time in the system among all work conserving disciplines that do not take advantage of precise knowledge of the job lengths, when the service time distribution has a decreasing hazard rate. More recently, Harchol et al. [4] have analyzed $L A S$ and show that for distributions with decreasing hazard rate $L A S$ effectively reduces the average time in the system with respect to $P S$, whereas it is the opposite for distributions with increasing hazard rate.

From the traffic point of view, it is known that Internet flow size distributions exhibit heavy tailed behavior (see, e.g. $[5,6,7]$ ) and can often be modeled by a distribution with a decreasing hazard rate, e.g. Pareto distribution. Thus most TCP sessions, e.g. interactive sessions, are of small size but a small amount of large flows, e.g. from data applications, are responsible for the largest amount of transferred data. As a consequence, if scheduling 
policies that favor short connections were to be implemented on the Internet, the average time in the system could be reduced.

Lastly, the perceived quality is more prone to high variations in transfer times of short flows than of long flows and, as we will argue, in the current TCP/IP network short transactions have wild variations in transfer times, in part due to the conservative value of the retransmission timer [8].

In several recent works $[9,10,11,12,13,14]$, the authors address the differentiation between short and long flows on Internet networks. In [9] the authors suggest two approaches based solely on simulation studies. The first approach is application based and it is proposed in the framework of Differentiated Services (DiffServ), with Assured Forwarding (AF) and RED with In and Out (RIO). This approach requires the non trivial choice of the numerous AF and RIO parameters. The second approach is TCP state based, using each connection's window size and relying on the compliance of the end hosts. Furthermore, this approach requires tuning of weighted round robin (WRR) parameters, which again is neither evident nor robust.

In $[10,11,12]$ the authors propose a two class based architecture to provide better service to short TCP flows. At the edge router, state information is kept for active flows. Packets are marked with high priority if the current length of the flow is below some threshold and inside the network service differentiation is performed by RIO routers or WRR scheduling. They present the gain obtained on mean response times through simulations. The results presented show reasonable gain in the average performance, but with no discussion on the worse case performance or on the variance of the performance. In $[11,12]$, the authors also discuss the analytical modeling of their approach, but are only able to give approximate numerical results based on the use of Kleinrock's conservation law [2].

In [13] and ensuing work [14] the authors study the $L A S$ (or $F B$ ) scheduling policy on the flow level and what the $L A S$ policy would produce in the context of a TCP network if packets from TCP flows were sorted in decreasing order of attained service.

The above mentioned papers have two main drawbacks, first of all the authors mainly rely only on one metric, the mean conditional response time for given flow size, to study sized-based differentiation mechanisms for TCP flows. We notice that dramatically different mechanisms such as Last Come First Serve $(L C F S)$ and $P S$ give identical mean conditional response times [2]. Furthermore, the performance metric for interactive applications is the latency, whereas throughput is a better performance metric for data applications.

We judge the effectiveness of size-based scheduling mechanisms for TCP flows not only on mean conditional response times but also on extreme values of response times. We also consider the system stability point of view, and study the number of ongoing connections. Through these performance measures, we are able to give a thorough and improved picture of the benefits of our scheme on the predictability of the performance of the flows and on the stability of the network.

Secondly, the proposed implementation mechanisms and simulation studies rely on buffer architectures requiring the tuning of many parameters, e.g. RIO and WRR. Although the use of simple priority queues with Drop Tail discarding can be argued to be too simple, it 
has the advantage of being robust and scalable. Furthermore, when assessing the usefulness of a novel differentiation scheme it is not evident from simulation studies if the differences in favor of the new scheme is due to the proposed way of marking the packets or due to the choice of scheduling parameters applicable only to the given simulation setting.

We propose a novel mechanism, RuN2C, as an implementation of a threshold based procedure, which does not necessitate state management nor packet tagging. This mechanism has the advantage of being implementable in different parts of a network: in access networks on the transmission side, in backbone networks (although authors have argued that such mechanisms are less useful in backbone networks) or more interestingly on the reception side of an access network. Also, the mechanism does not require prior agreements between ISPs to be used across networks and is robust to small variations, e.g. orders of 10 packets in the value of the threshold.

We derive asymptotical results based on analytical models of the proposed mechanisms, assuming tail drop routers, to show that threshold based mechanisms produce close to standard TCP transfer times for large transfers while $L A S$ may discriminate severely against large transfers. Furthermore, we show that by an optimal choice of the threshold, the mean response time in the system can be chosen close to that of $L A S$. The novel threshold based mechanism, RuN2C, is then compared to $L A S$ based mechanisms and standard Drop Tail scheduling through simulations. We show that RuN2C produces an important improvement of performance with respect to all cited metrics for short flows, while treating large flows as the current TCP implementation does.

We will use the following terms. We will call RuN (Running Number differentiation mechanism) an implementation of $L A S$ over a TCP/IP network, where TCP packets are given priority according to the rank of their first data byte in the connection's data stream. We will call RuN2C (Running Number 2 Class differentiation mechanism) an implementation over a TCP/IP network of a two class scheduling mechanism in which packets are classified as high or low priority according to the comparison of the packet's first data byte rank in the connection's data stream with a given threshold.

The rest of the paper is organized as follows. Section II discusses the theoretical justifications for the RuN2C mechanism in comparison to standard TCP over Drop Tail queues and to $L A S$. Section III provides details for a possible implementation of RuN2C in a TCP/IP network. Section IV provides a more exhaustive study of RuN and RuN2C via simulations. In particular, we study the variability of TCP transfers' response times. The paper is concluded in section $\mathrm{V}$.

\section{Algorithms and Mathematical Models}

In this section we analyze two packet level scheduling policies for the differentiation between short and long TCP flows. We study these scheduling policies using $P S$-type flow level models. In particular, we are interested in calculating the mean response time $\bar{T}(x)$ for jobs with service time $x$. In order to evaluate the impact that giving priority to short connections has on large flows, we also study the asymptotical value of $\bar{T}(x)$ as $x \rightarrow \infty$. The notation with 
a suppressed dependence on $x$ is used to denote the overall mean response time. The other metrics of interest are the throughput, $\Theta(X)=x / T(x)$, and the slowdown, $S(x)=T(x) / x$. From the user perception, the mean response time and its variance is an appropriate metric for the HTTP type web traffic and the average throughput is an appropriate metric for the FTP type traffic. We study these metrics in more detail in Section IV.

Assuming a Poisson flow arrival process, a small Bandwidth Delay Product (BDP), and comparable Round Trip Times (RTTs), the current realization of the TCP/IP network is well represented by the $M / G / 1-P S$ queuing model $[5,15]$. The performance metrics for the $M / G / 1-P S$ model with the capacity scaled to one is given by [2]

$$
\begin{gathered}
\bar{T}^{P S}(x)=\frac{x}{1-\rho}, \\
\bar{\Theta}^{P S}(x)=1-\rho, \\
\bar{S}^{P S}(x)=\frac{1}{(1-\rho)} .
\end{gathered}
$$

Based on these performance metrics, the Processor Sharing policy is fair in the sense that it gives a constant throughput to all flows regardless of their size. Thus the mean slowdown of the flow is also independent of the flow size.

\subsection{RuN Scheduling Policy}

We first analyze the $L A S$ flow level scheduling policy and its packet level implementation RuN. For the $L A S$ implementation one should know the running number of the packet, i.e., one should know if the packet is the first, third, tenth, etc. packet of a flow. The RuN packet level scheduling policy consists in serving the packets arriving to the router buffer in ascending order according to their running number. Hence, the abbreviation for this implementation scheme is RuN. On the flow level, the $L A S$ or Foreground Background $\left(F B_{\infty}\right)$ scheduling policy has been thoroughly studied (see e.g. [2] $)^{1}$.

Let us consider a flow that requires a total service time of $x$. Let $F(x)$ be the distribution function of the flow service times. Next we denote as $\overline{X_{x}^{n}}$ the $n$-th moment of the truncated distribution at $x$. Namely,

$$
\overline{X_{x}^{n}}=\int_{0}^{x} y^{n} d F(y)+(x)^{n}(1-F(x)) .
$$

The utilization factor for the truncated distribution is $\rho_{x}=\lambda \overline{X_{x}^{1}}$. This value represents the virtual load a customer of size $x$ sees in the system. From [2] we know that the average response time conditioned on the flow size is

$$
\bar{T}^{L A S}(x)=\frac{\bar{W}(x)+x}{1-\rho_{x}}
$$

\footnotetext{
${ }^{1}$ For the sake of analytical tractability we use here the model with an infinitesimal size of the service quanta given to each user. But the results can be extended to the case of finite service quanta $[16,17,18]$.
} 
with

$$
\bar{W}(x)=\frac{\lambda \overline{X_{x}^{2}}}{2\left(1-\rho_{x}\right)} .
$$

The $L A S$ scheduling policy outperforms the $P S$ scheduling policy in terms of the overall mean response time if the service time distribution has a decreasing hazard rate [4]. In such a case we have $\bar{T}^{L A S} \leq \bar{T}^{P S}$, even though some large flows suffer (see Figure 2). Furthermore, if the flow size distribution has finite mean and variance, then, as shown in [19],

$$
\lim _{x \rightarrow \infty} \bar{S}^{L A S}(x)=\lim _{x \rightarrow \infty} \bar{S}^{P S}(x)=\frac{1}{1-\rho} .
$$

Specifically, consider the Pareto distribution, $F(x)=1-(k / x)^{\alpha}$, which is an acceptable approximation for the file size distribution for the current Internet state. Clearly, its hazard rate,

$$
\mu(x)=\frac{F^{\prime}(x)}{1-F(x)}=\frac{\alpha}{x}
$$

is a decreasing function, and thus $\bar{T}^{L A S} \leq \bar{T}^{P S}$.

Let us analyze the asymptotics for $\bar{T}^{\bar{L} A S}(x)$ as $x$ goes to infinity. From expression (4) one immediately obtains that

$$
\bar{T}^{L A S}(x)=\frac{1}{1-\rho} x+\frac{\lambda \overline{X^{2}}}{2(1-\rho)^{2}}+\mathrm{o}(1) .
$$

We note that if the variance $\overline{X^{2}}$ is large, the mean conditional response times for the middle size flows in the case of $L A S$ can be significantly larger than in the case of $P S$. Moreover, if the variance is infinite $(1<\alpha<2)$, the asymptotics has the following form

$$
\bar{T}^{L A S}(x)=\frac{1}{1-\rho} x+\frac{k^{2+\alpha}}{2-\alpha} x^{2-\alpha}+\mathrm{o}\left(x^{2-\alpha}\right) .
$$

There is no asymptote in this case, even though the $\operatorname{limit}_{\lim } \rightarrow \infty \bar{S}^{L A S}(x)$ exists. This implies that the performance of $L A S$ deviates increasingly from $P S$ performance with the increase of the file size.

Finally, we note that the $L A S$ scheduling policy can be very unfair. Consider the case when many flows of the same size arrive subsequently in such a manner that a new flow enters the system just before the previous flow is about to be finished. In this scenario, all these flows will leave the system at the same time under the $L A S$ policy. There might be a huge difference between the response times for the first and the last flows. On contrary, the $P S$ scheduling discipline will treat all flows fairly in this scenario.

The above remarks and the difficulty in implementing the RuN mechanism prompt us to look for some scheduling policy which combine good properties of both $L A S$ and $P S$. An example of such a policy is proposed in the next section. 


\subsection{RuN2C Scheduling Policy}

RuN2C is a threshold based scheduling policy that blends the good features of both $L A S$ and $P S$. On the flow level, this policy serves flows in the first queue as long as they have received an amount of service less than a given threshold. They then move to the second queue, which is serviced only if the first queue is empty. Inside the queues flows are serviced using the $P S$ discipline. This policy corresponds to the two level $P S$ scheduling policy $M / G / 1-P S+P S$ introduced in [2]. On the packet level, we consider a drop tail queue with a threshold based two class priority mechanism, hence the abbreviation RuN2C for the packet level mapping. Upon a packet arrival in a router, the running number is inferred and is compared to a given threshold value. The packet is marked to either a high priority with small running number or a low priority with high running number. The packets with a number smaller than the threshold (class 1 packets) will be serviced before the packets with a number larger than the threshold (class 2 packets). A possible implementation is presented in Section 3.

Let $t h$ be the value of the threshold. Given a flow of size $x$ where $x>t h$, the first $t h$ packets of a flow will have high priority, while the rest $x-t h$ packets will be considered as low priority packets. ${ }^{2}$ For those flows with a size equal or smaller than $t h$, the system behaves as a pure $P S$ system where the service time distribution is truncated at $t h$. Hence the mean response time is given by

$$
\bar{T}^{P S+P S}(x)=\frac{x}{1-\rho_{t h}},
$$

for $x \in[0, t h]$. The average number of flows served during a busy period is $1 /\left(1-\rho_{t h}\right)$. For flows with size $x \in(t h, \infty)$ the mean response time conditional on the flow size is given by

$$
\bar{T}^{P S+P S}(x)=\frac{\bar{W}(t h)+t h+\alpha(x-t h)}{1-\rho_{t h}} .
$$

The expression consists of the delay due to the time spent in the first high priority queue, where the flow is serviced up to the threshold $t h,(\bar{W}(t h)+t h) /\left(1-\rho_{t h}\right)$, and the time spent in the lower priority queue $\alpha(x-t h) /\left(1-\rho_{t h}\right) . \bar{W}(t h)$ is given by (5).

The only unknown expression is $\alpha(x)$. It is the virtual time spent in the lower priority queue and to solve it one can consider a queuing system with bulk arrivals to the lower priority queue after a busy period in the high priority queue. This approach results in the following integral equation [2] for $\alpha^{\prime}(x)=\mathrm{d} \alpha(x) / \mathrm{d} x$

$$
\begin{aligned}
\alpha^{\prime}(x)= & \lambda \bar{n} \int_{0}^{\infty} \alpha^{\prime}(y) B(x+y) d y \\
& +\lambda \bar{n} \int_{0}^{x} \alpha^{\prime}(y) B(x-y) d y \\
& +b B(x)+1,
\end{aligned}
$$

\footnotetext{
${ }^{2}$ We remind that here we consider the normalized service time.
} 
where

$$
\bar{n}=\frac{1-F(t h)}{1-\rho_{t h}}
$$

is the mean fraction of flows that reach the low priority queue after a busy period of the high priority queue,

$$
b=2 \lambda(1-F(t h)) \frac{\bar{W}(t h)+t h}{1-\rho_{t h}}
$$

is the average number of flows that arrive to the low priority queue conditioning that at least one does. $B(x)$ is the complementary truncated distribution given by

$$
B(x)=\frac{1-F(t h+x)}{1-F(t h)},
$$

which for the exponential case, due to the memoryless property, reduces to the form given in $[2], B(x)=1-F(x)$.

The integral equation (7) can be solved by using either the finite approximation of the Riemann sum or fixed point iterations. Note that though in [12] the authors use the same approach to model their system, and derive a similar integral equation, they are not able to solve the resulting integral equation, even numerically.

Theorem 1 describes the limiting behavior of the slowdown for large file sizes in the case of $M / G / 1-P S+P S$ scheduling policy.

Theorem 1 Let the service time distribution have a finite mean. Then the slowdown for the PS +PS scheduling policy has a limit as the flow size goes to infinity

$$
\lim _{x \rightarrow \infty} \bar{S}^{P S+P S}(x)=\lim _{x \rightarrow \infty} \bar{S}^{P S}(x)=\frac{1}{1-\rho} .
$$

Furthermore, the mean conditional response time $\bar{T}^{P S+P S}(x)$ has an asymptote with the slope $1 /(1-\rho)$ if $2 \rho-\rho_{\text {th }}<1$.

The proof of the theorem is postponed to the Appendix.

Based on Theorem 1 we note that the advantage of the $P S+P S$ scheduling discipline against $L A S$ is that there exists an asymptote for $\bar{T}^{P S+P S}(x)$ as $x$ goes to infinity even in the case of file sizes distributed with an infinite variance, as is the case for the Internet file size distributions. Thus, the absolute difference between $P S+P S$ and $P S$ transfer times remains bounded. As a consequence, very large flows should not see a difference between RuN2C and the current TCP/IP implementation.

We study numerically the value of the mean response time, $\bar{T}^{P S+P S}$. Since $P S+P S$ gives priority to short jobs, $P S+P S$ acts in the same way as $L A S$ reducing the overall mean response time compared to $P S$, when the hazard rate of the service time distribution is decreasing [4]. In Figure 1 we depict the value of the average response time as a function 


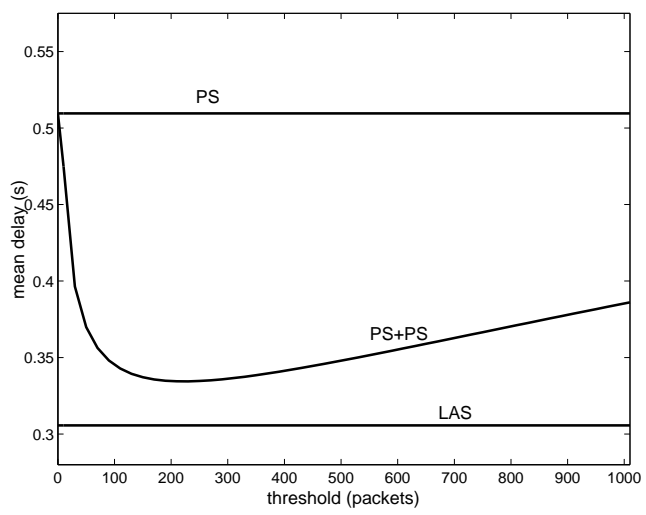

Figure 1: Optimal value of the threshold for $\bar{T}^{P S+P S}$ for a heavy tailed flow size distrinution.

of the threshold for a bounded Pareto flow size distribution with parameters $B P(k, p, \alpha)=$ $B P(13,5000,1.1)$ and mean 64 packets. An optimal choice of the threshold in the $P S+P S$ scheme gives nearly the same gain in overall performance as in the case of $L A S$, without the drawbacks of servicing very long flows unfairly. This result is even more striking if we keep in mind that $L A S$ is optimal with respect to the average time in the system, when the hazard rate of the service distribution is decreasing, among all the work conserving disciplines that do not take advantage of precise knowledge of the job lengths [3]. Of course, we recognize that the optimal theoretical value of the threshold may be different from the value which gives the best performance for a real implementation. For a Pareto distribution, with maximum flow size of 5000 packets, the optimal threshold is around 200 packets, showing that choosing the threshold as the minimum value for the TCP congestion control to avoid retransmits for small flows, see Section 3 , is conservative and penalizes long flows less severely than $L A S$.

Interestingly, the benefit that $L A S$ and $P S+P S$ provide to short flows provokes a smaller degradation of the performance for large flows in the case of $P S+P S$ than in $L A S$. Figure 2 shows the delay for flows for the three different scheduling policies, as a function of their size. The flow size distribution is bounded Pareto with parameters $B P(13,5000,1.1)$ and mean 64 packets. We observe that even though large flows do not suffer much with $P S+P S$, the average time in the system is reduced significantly (see Figure 1).

The analytical flow level models presented in this section can be used to model the proposed packet level implementations, as long as the transient effect of TCP congestion control, namely the initial slow start phase is taken into account. Thus, assuming that the TCP maximum window size is the bottleneck, not the BDP of the link and looking at the cycle for every RTT and assuming that an application sends one window, $w$, worth of packets one packet at a time during one RTT, we have

$$
\bar{T}^{S S}(x)= \begin{cases}\left(\left\lfloor\log _{2} x\right\rfloor+1\right) R T T, & x \leq 2^{\left\lceil\log _{2} w\right\rceil+1} \\ \bar{T}_{S S}(x-1)+R T T / w, & \text { otherwise. }\end{cases}
$$




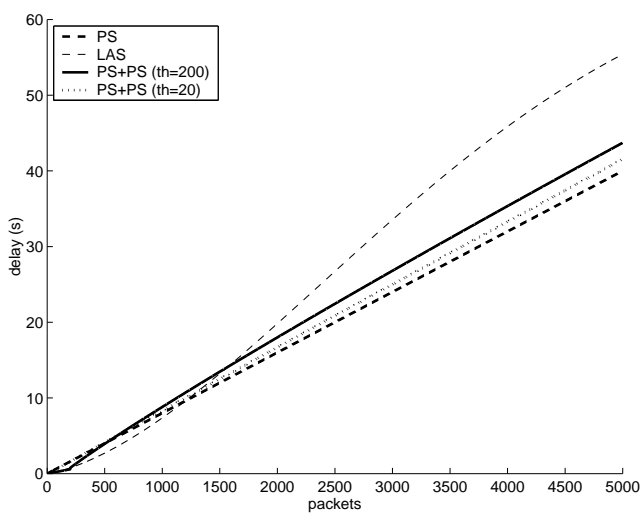

Figure 2: Difference in delay as a function of flow size for a heavy tailed flow size distribution.

Adding the slow start model to the scheduling delay models of this section have a good correspondence with the simulation results of Section 4. Due to limited available space, we only consider the simulation results, but conclude that the delay of TCP flows can be modeled by two main components: slow start and queueing.

\section{Implementation of RuN2C for TCP/IP}

As explained previously, RuN2C is a threshold based two class differentiation mechanism. The packet level implementation requires maintaining a two class priority queue and knowledge of the value of the threshold parameter between high and low priority queues. As we are giving priority to flows amounting to a small network load, there is no risk of the priority queue starving the lower priority queue and thus little need for extra weight parameters for the queues.

In order to propose a stateless implementation, routers must be able to classify packets from a TCP connection without taking into account previous events. To this end we propose that routers infer the served amount of bytes by only looking at the current TCP sequence number ${ }^{3}$. TCP sequence numbers are incremented from one segment to the other by the number of bytes in the packet's workload and the initial sequence number is picked at random when the TCP connection is established.

The principal retained is to have TCP sequence numbers start from a set of Possible Initial Numbers $P I N_{i}$ where $i \in\left\{1, \ldots, 2^{R}\right\}$ equally spaced in the sequence number field ranging from 0 to $2^{32}-1$. These numbers should be spaced not too far to allow for the initial

\footnotetext{
${ }^{3}$ As this may be inefficient in terms of packet processing in routers, this lookup may also be performed in edge routers. These may then set TOS bits in the IP header if the mechanism is to be used in routers which may not allow for sequence number lookup.
} 
sequence number of a TCP connection to be picked sufficiently at random ${ }^{4}$. They must be spaced far enough to reduce the probability (or the occurrence rate) of running over to the next PIN.

Let $t h$ be the value in bytes of the threshold, packets for which the sequence number is between $P I N_{i}$ and $P I N_{i}+t h$ will be classified as priority packets in contrast to packets for which the sequence number lies between $P I N_{i}+t h$ and $P I N_{i+1}$, where $P I N_{i+1}$ is the next possible initial number (see Figure 3 ).

With this structure the sequence number expressed in binary code is divided into three parts (see Figure 3). The $R=32-(L+T H)$ most significant bits are picked at random, providing $2^{R}$ different PIN values. The next $L$ bits and the following $T H$ bits, where $T H=\log _{2} t h$, are set to zero when the TCP connection is established. This scheme permits to infer the priority of the packet by a simple mask based comparison, since when the sequence number belongs to the low priority range $\left[P I N_{i}+t h, P I N_{i+1}\right]$ the $L$ intermediate bits will be equal to 0 . Packet sequence numbers from a given connection will overflow to the next $P I N$ after $2^{L+T H}$ bytes which we show in the Subsection 3.1 can be chosen quite large.

Note that since the sequence number is counted in bytes, the interval $2^{T H}$ is divided into $M S S$ (Maximum Segment Size) disjoint sets. This allows us to choose also at random the first $\log _{2} M S S$ bits of the initial sequence number. We can thus increase the randomness of the scheme allowing both the first $R$ bits and the $\log _{2} M S S$ least significant bits to be random.

As our implementation retains the per byte nature of TCP flow control, the number of high priority bytes of each connection will be fixed and independent of the $M S S$ of the connection. In packet count based schemes, e.g. [10], the number of high priority segments is fixed and the number of high priority bytes depends on the MSS. Our scheme provides positive features from both the user and network point of view. From the network point of view, the maximum number of segments per connection that will get high priority can be controled just by setting the values of $T H$ based on a small data segment (typically 512 bytes). From the user point of view, since the number of bytes with high priority will be fixed by the network, this scheme is transparent and will guarantee a well-defined fairness with respect to other users and at the same time it will prevent misbehaving users from getting preferential treatment since whatever the value of $M S S$ a connection choses, only the number of bytes defines the amount of preferential treatment obtained.

\subsection{Dividing the sequence number space}

The threshold $t h$ must be chosen in such a way that short flows benefit from the differentiation mechanism while keeping the load of high priority low in order not to harm longer flows. To find a compromise solution, we note two facts. First, short TCP flows are prone to timeouts upon packet losses (see for example [21]). The impact of timeouts can be extremely important on the response time of short flows since its minimum value is $1 \mathrm{~s} \mathrm{[8].} \mathrm{Thus,} \mathrm{to}$

\footnotetext{
${ }^{4}$ Misbehaving users should not be able to infer the initial sequence numbers [20].
} 


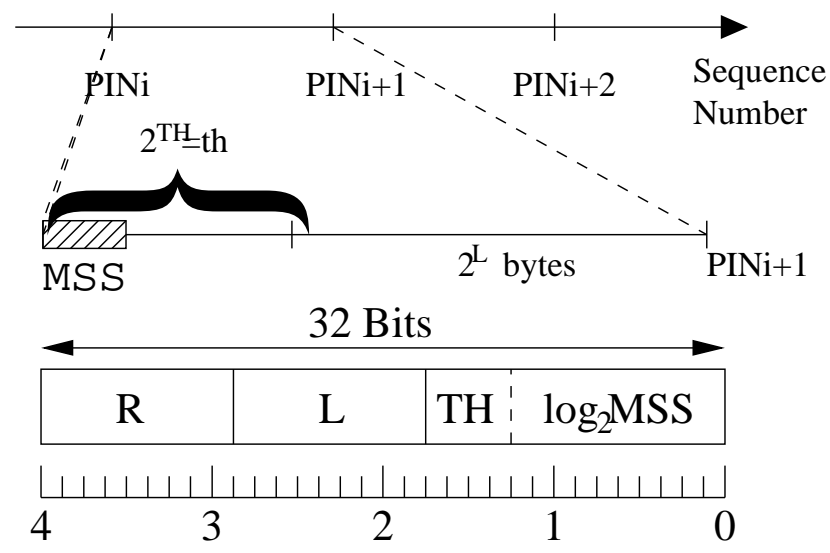

Figure 3: Structure of the sequence number for RuN2C

avoid timeouts, packets should be given priority until the congestion window reaches a value of 3 or 4 . This is the case if approximately 8 packets are transmitted. This corresponds to a threshold $t h$ of $12 \mathrm{KBytes}$ if $M S S=1460 \mathrm{KBytes}$. Second, since TCP flow sizes are heavy tailed, we may expect for flows shorter than 8 packets to account for a limited proportion of the total load.

To allow for $t h=12 k$ Byte we require $T H=\log _{2}(12000) \approx 14$ bits for the least significant bits in the sequence number. Let us consider $L$ to be 8 . Sequence numbers will overflow to the next PIN after having sent $2^{22} \approx 4$ Mbytes. The latter amount of data corresponds to 2800 packets. At the worst case, this might induce a packet loss with probability $3.6 \times 10^{-4}$, which is negligible in respect to the current packet loss rate in the Internet. With this setting there are $2^{10}=1024$ possible values for $P I N$. This may not provide satisfactory degree of randomness in the choice of the initial sequence numbers. As mentioned previously, this can be improved choosing the first $\log _{2} 1460 \approx 10$ bits at random. Therefore, the total number of random bits available is equal to $10+10=20$, which can produce 1 million random values.

\subsection{Deployment}

We now give some indications on deploying such a mechanism in a TCP/IP network. Standard TCP connections may be sharing RuN2C enabled routers. In such a case they will not benefit from the priority queue except if they randomly start their sequence number to do so. Analytical models in the previous section and the simulation results given in the next section, show that long TCP connections obtain equivalent performances with tail drop routers and $\mathrm{RuN} 2 \mathrm{C}$ routers as long as the load of the priority traffic remains small. One may expect for a similar result to stand for non-adapted short TCP flows if the priority traffic is sufficiently small. RuN2C routers can thus be considered compatible with current 
TCP implementations. In addition TCP connections implementing RuN2C are not affected by tail drop routers. We thus conclude, that RuN2C can be progressively deployed on a network, as it is always beneficial for all adapted TCP connections and never worse than current for non-adapted connections.

\section{Simulations}

The two packet level scheduling disciplines (RuN and RuN2C) presented in the previous section were studied analytically on the flow level using $P S$-type models. In this section we use the NS simulator [22] to study metrics which were not analyzed in the Section II, i.e., the extreme values of the response times and the number of ongoing flows in the system. In NS flow sequence numbers start from zero for each flow and the implementation of RuN and RuN2C schemes is straightforward.

The simulation topology used consists of a 10Mbps bottleneck link serviced by 10 access links. The bottleneck buffer has a finite size of 100 packets. We use two basic, but different simulation settings:

(S1) 10Mbps access links with a total network propagation delay of $6 \mathrm{~ms}$.

(S2) $2 \mathrm{Mbps}$ access links with a total propagation delay of $60 \mathrm{~ms}$.

(S1) is chosen to be one where the arrival process to the bottleneck link is bursty and a single connection may completely use the bottleneck capacity. Thus the traffic in the network will experience many losses and longer queuing delays. In (S2) the arrival process is less bursty, due to the smaller bandwidth of the access links, and the delay due to propagation delay is more dominant than the delay due to queuing. In all simulations, packet size is set to 1500 bytes and maximum TCP window is 30 packets. We use in RuN2C a threshold of 20.

In the simulations, the schemes are implemented by inspecting the sequence number of the packet from the TCP header at the bottleneck router. The packet is then placed in the buffer either in ascending order according to its sequence number (RuN) or classified to higher or lower priority level (RuN2C) based on a predefined sequence number threshold. In both scheduling mechanisms, the tail drop is in a push out fashion, i.e. if the buffer is full, the arriving packet is first placed into the buffer, and then the tail of the buffer is pushed out. The two proposed scheduling mechanisms are then compared to a traditional FIFO Drop Tail queue.

We consider two flow size distribution scenarios:

(D1) A heavy tailed distribution, which is a mixture of two distributions. $80 \%$ of flows are short TCP flows with exponentially distributed flow sizes with mean size of 4 packets ( 6 kbytes) and $20 \%$ are long TCP flows with a bounded Pareto distribution $B P(k, p, \alpha)=B P(13,5000,1.1)$ and mean 64 packets. Thus the load of short flows is $20 \%$ and the load of long flows $80 \%$. 
(D2) 50 persistent TCP flows and a $20 \%$ load of short TCP flows with exponentially distributed flow sizes with mean size of 6 packets (9 kbytes).

We use the following metrics to assess the performance of the different mechanisms:

- number of flows in the network,

- mean and maximum latency for short TCP flows,

- throughput and its variance for long TCP flows.

\subsection{Mixed distribution of short and long flows}

Consider topology (S1) with traffic distribution (D1). In Figure 4 we plot the number of flows in the system under the three different schedulers as a function of time, thus depicting the change in the stability of the network. RuN and RuN2C mechanisms are able to considerably

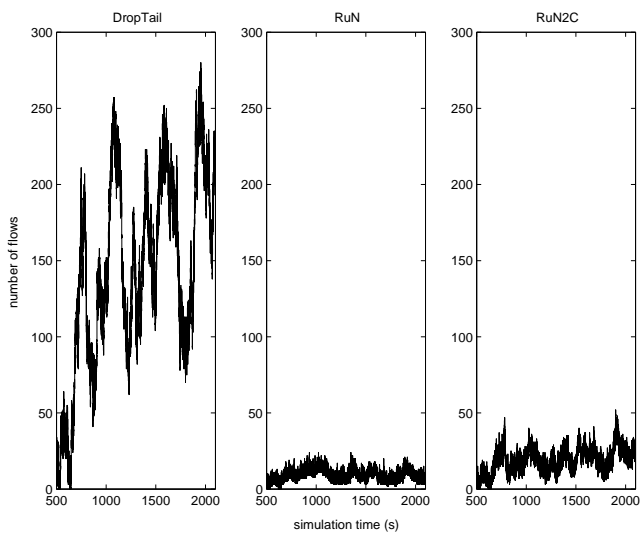

Figure 4: Number of flows in the network during the simulation under setting (S1) and distribution (D1)

reduce both the average number of flows in the network as well as the variability. From the figures we thus notice that RuN2C is nearly as efficient as RuN to reduce the average overall number of flows.

Figure 5 shows the change in delay under the three schedulers. We can make two observations from the figure. The mean response time is reduced using the RuN and RuN2C schedulers by more than a ten fold for all flows under the threshold of 20 packets and considerably for flows less than 40 packets i.e. $60 \mathrm{kbytes}$. Thus the proposed scheduling mechanisms are able to reduce the delay for the short flows. Figure 5 also nicely illustrates the difference between the two RuN schemes. Up to the threshold of 20 packets there is minimal difference in strictly ordering the packets and in merely dividing them into two classes. After 


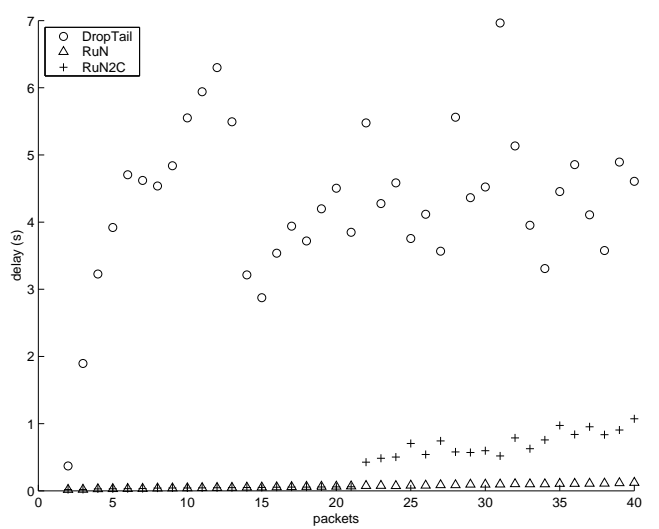

Figure 5: Mean delay as a function of packet size for short flows under setting (S1) and distribution (D1)

the threshold the RuN2C scheme resembles the DropTail though for flow sizes close to 20 packets the effect of the preferential treatment to the first 20 packets is still visible in a reduced delay.

Besides the average delay, we study the maximum delay for the short flows shown in Figure 6 . We notice that under the RuN and RuN2C schemes the maximum delay is close to the mean delay for all short flows, while the DropTail scheme results in some flows having very large delays. As an example consider the flow with size of 7 packets, under the RuN and RuN2C schemes it has a maximum delay of $100 \mathrm{~ms}$, while under DropTail the maximum delay is $200 \mathrm{~s}$, a difference of 3 orders. This is the result of multiple backoffs of the TCP retransmission timer. This example illustrates the blackout impression users can have even in a moderately loaded system. Thus, RuN and RuN2C schemes significantly reduce the timeouts for short flows and hence improve the predictability of response times.

Figure 7 depicts how the gain in smaller delay for the short flows affects the throughput of long flows. It seems that for a maximum flow size of 5000 packets i.e. 7.5Mbytes, all flows have the same or better throughput under the RuN and RuN2C schemes compared to DropTail. However, as will be shown later the RuN scheme may not be beneficial for all long flows if many long flows coincide at the same time.

Consider now topology (S2), with smaller access link capacities and a larger overall RTT. It is expected that the gain in smaller delay for the short flows is not as considerable as there are fewer drops and thus fewer retransmits in topology (S2), furthermore the propagation delay dominates the total delay for flows. Figure 8 depicts the number of flows in the network. Though the aggregated packet arrival process to the bottleneck link is less bursty, there is still a difference in the stability of the network. 


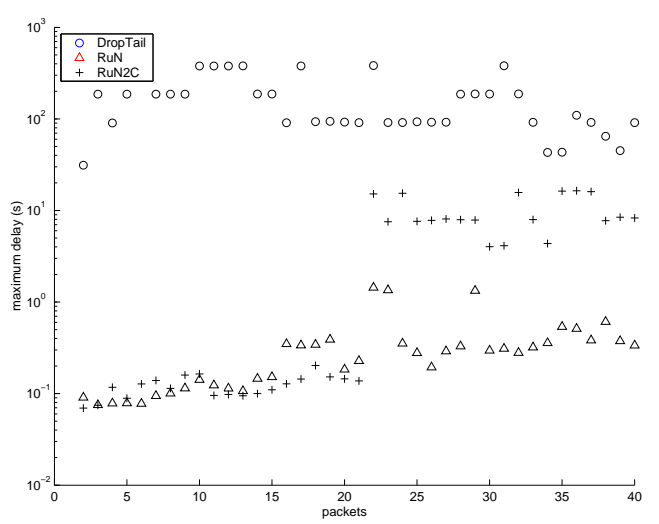

Figure 6: Maximum delay as a function of packet size for short flows under setting (S1) and distribution (D1)

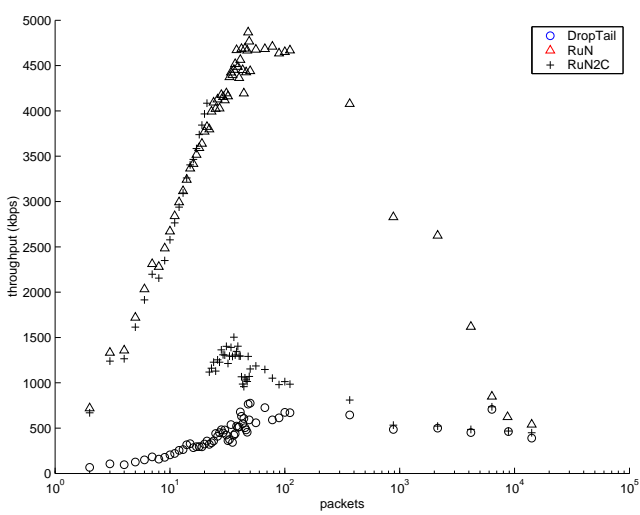

Figure 7: Mean throughput as a function of packet size for all flows under setting (S1) and distribution (D1)

Figure 9 shows the mean response time using the two schedulers. Though the RuN and RuN2C schemes are able to reduce the delay, the effect of slow start (step like pattern) and the large propagation delay are dominant.

We notice that the change in average delay is still considerable. Mainly we notice that the delay under DropTail is of the same magnitude for a propagation delay of $6 \mathrm{~ms}$ or $60 \mathrm{~ms}$. While for RuN and RuN2C schemes the delay is clearly mainly due to propagation delay and marginally due to queuing delays. The maximum delay is also reduced for the short flows, as shown in Figure 10.

$\mathrm{RR} \mathrm{n}^{\circ} 4964$ 

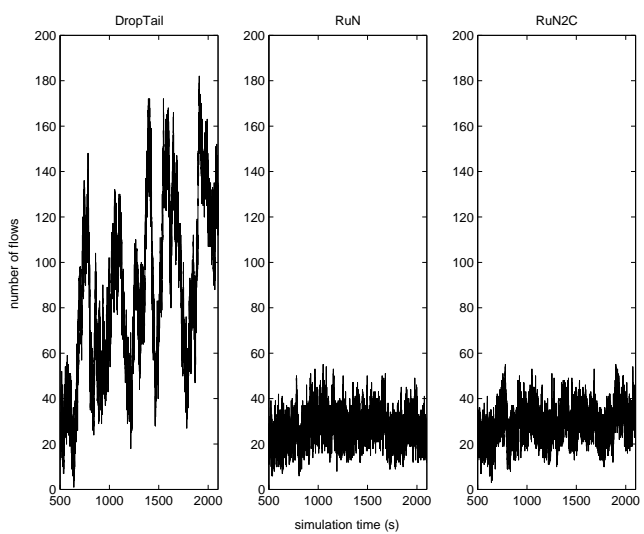

Figure 8: Number of flows in the network during the simulation under setting (S2) and distribution (D1)

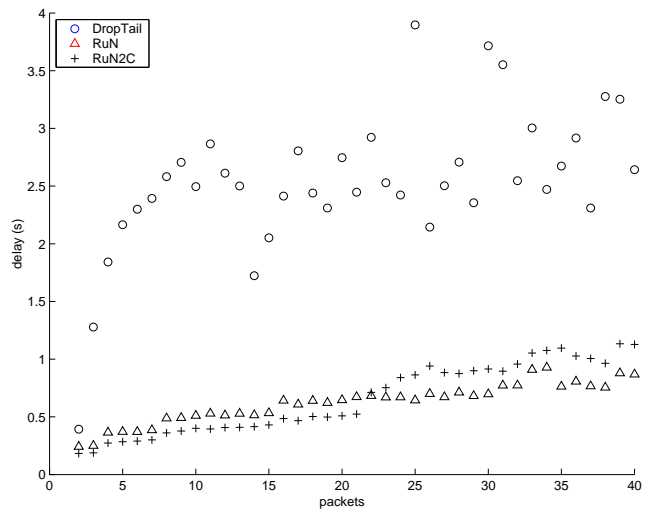

Figure 9: Mean delay as a function of packet size for short flows under setting (S2) and distribution (D1)

Figure 11 depicts that as the change in delay for the short flows was not as large, neither is the change in throughput for the long flows. However, it still seems that the RuN2C scheme is able to improve the throughput for all flows.

\subsection{Effect on persistent flows}

Now consider the same two network parameter settings as previously, but the flow distribution (D2). Instead of long TCP flows that arrive randomly with a fixed size we load the network with 50 persistent TCP flows. We then study how the scheduling mechanisms affect 


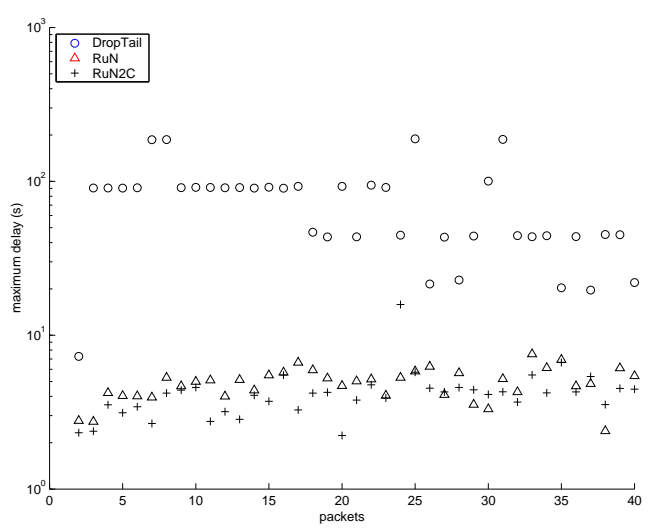

Figure 10: Maximum delay as a function of packet size for short flows under setting (S2) and distribution (D1)

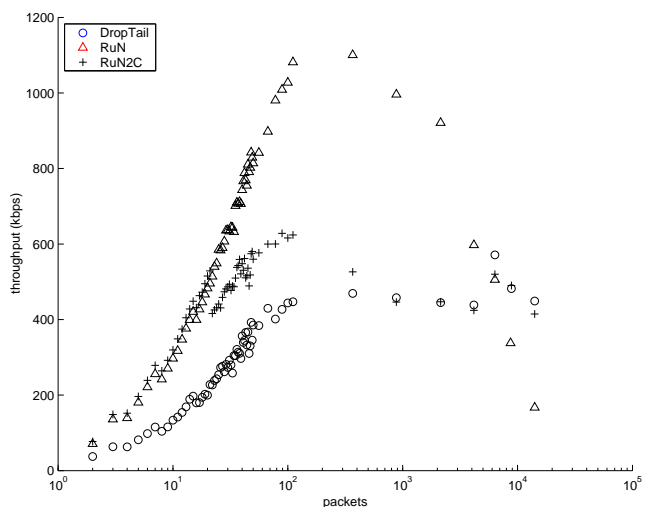

Figure 11: Mean throughput as a function of packet size for all flows under setting (S2) and distribution (D1)

these persistent flows and the randomly arriving short flows that coexist with the persistent flows. We present the same set of metrics as in the previous section. The effect of the RuN schemes on the persistent flows is different than on the randomly arriving long flows. However, studying the persistent flows gives us an indication of the effect of scheduling during a snapshot of the network, when long flows coexist at the same time.

Figure 12 depicts the number of flows in the network on the log scale, as the reduction in the number of short flows is of more than two orders.

Figure 13 shows the change in average delay for short flows under the three schedulers. The stabilizing effect of the RuN schemes is clear from figures 12 and 13. Under DropTail 


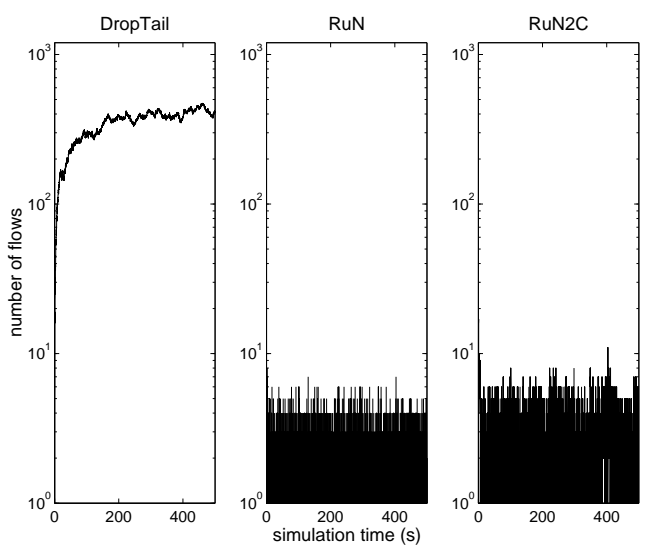

Figure 12: Number of short flows in the network during the simulation under topology (S1) and distribution (D2)

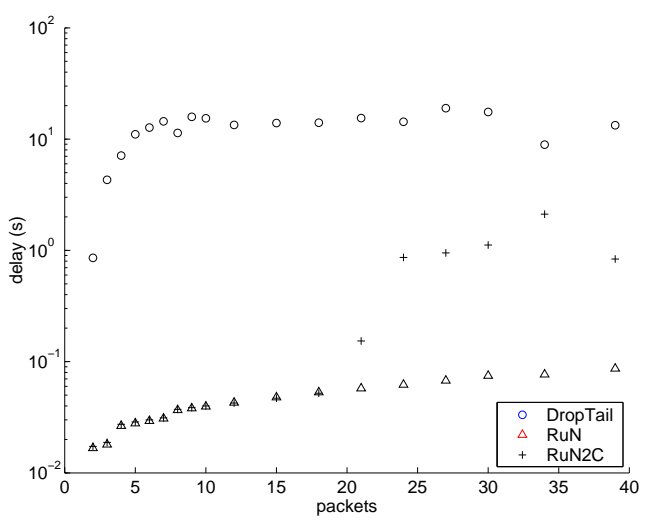

Figure 13: Mean delay as a function of packet size for short flows under topology (S1) and distribution (D2)

the latency for short flows is dominated by drops and retransmits as it has been already mentioned in Section 4.1.

Figure 14 depicts how the gain in smaller delay for the short flows affects the throughput of persistent flows. From the figure we see that the 50 persistent flows have negligible difference between the mean throughputs, though under RuN the throughput is the lowest. There are, however, large differences in the variance of the throughput. The RuN scheme reduces the mean throughput and has zero variance. This is because the RuN scheme services the persistent flows exactly the same amount and as a consequence the data points 


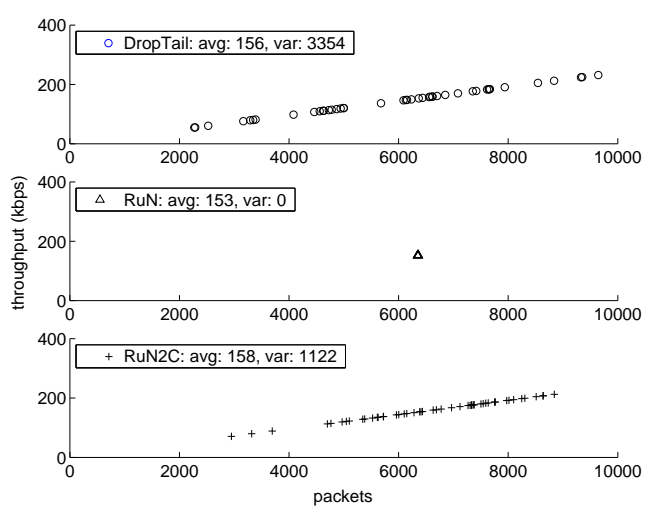

Figure 14: Mean throughput as a function of packet size for persistent flows under topology (S1) and distribution (D2)

in the middle figure all coincide and look like one data point! Under RuN, all the persistent flows start at the same time and are serviced in the order of packet sequence numbers in a strict round robin fashion packet by packet. If at some point a more aggressive flow has more packets serviced than others, it has to wait in the buffer or is pushed out of the buffer and thus slowed down until the others have caught up with the aggressive flow. This shows how the $L A S$ policy may reduce the overall mean response time if all long flows start at the same time, but may be extremely unfair for long flows starting during the service time of other long flows, as discussed in Section II.

Note also that the throughput variance for RuN2C is three times smaller than the throughput variance for DropTail. The RuN2C scheme is thus able to both increase the average throughput as well as reduce the variance, without the risk of the pathological behavior of $L A S$. For the topology (S2) the conclusions are the same and figures are omitted due to lack of space.

\section{Conclusions}

We propose a packet level size-based scheduling mechanism for TCP flows, RuN2C, which is a threshold based differentiation scheme. We describe an implementation of this mechanism which has the advantage of being TCP compatible, robust, scalable and progressively deployable. We show numerically, how the value of the threshold affects the optimality of the scheduling policy and compare by means of simulations the proposed implementation to standard TCP over tail drop buffers and to $L A S$ implementation RuN.

We showed that RuN2C is beneficial from both the system's and the user's point of view for small transfers. From the user's perspective the network response becomes more predictable and the average response time is reduced. In addition, many short flows get 
served promptly at the expense of some few long flows. In fact, we show that RuN2C performs very similar to standard TCP for large flows. From the system's point of view, the average number of active sessions is reduced.

On the contrary, we show that $L A S$ based implementations, on top of being difficult to implement, may show degraded performance for large transfer sizes since for service distributions with infinite variance $L A S$ deviates increasingly from $P S$ for large file sizes. This is in contrast to RuN2C, which exhibits performance close to that of standard TCP for large transfers and is simpler to implement. At the flow level, we show by numerical analysis that the $P S+P S$ scheduling policy reduces the mean response time $\bar{T}^{P S+P S}$ with respect to $P S$ when the hazard rate of the service time distribution is decreasing. In addition, we provide indications that by an appropriate tuning of the threshold, $P S+P S$ performs very closely to $L A S$, which is known to be the optimal scheduling discipline, with respect to the average time in the system, among work conserving disciplines, when no precise knowledge of the job lengths is available and when the hazard rate of the job distribution is decreasing.

At the TCP level the benefit brought by the RuN2C mechanism seems to come at no cost. This benefit is obtained as a consequence of a better work scheduling, from the performance point of view, of the loss epochs. That is, short TCP flows are extremely vulnerable to packet loss and they have a high probability of experiencing a costly timeout. In contrast, large TCP flows are more robust against losses since they are more likely to have large congestion windows. Therefore, many short sessions benefit from giving priority to short flows at almost no cost for long flows, as short sessions account for a small proportion of total network load.

\section{References}

[1] N. Bhatti, A. Bouch, and A.J. Kuchinsky, "Integrating user-perceived quality into web server design," in Proceedings of WWW'00, Amsterdam, The Netherlands, 2000.

[2] Leonard Kleinrock, Queueing Systems, vol. 2, John Wiley and Sons, 1976.

[3] S.F. Yashkov, "Processor-sharing queues: Some progress in analysis," Queueing Systems, vol. 2, pp. 1-17, 1987.

[4] A. Wierman, N. Bansal, and M. Harchol-Balter, "A note on comparing response times in the $\mathrm{M} / \mathrm{G} / 1 / \mathrm{FB}$ and $\mathrm{M} / \mathrm{G} / 1 / \mathrm{PS}$ queues," Tech. Rep. CMU-CS-02-177, Carnegie Mellon University Research, Sept. 2002.

[5] S. Ben Fredj, T.Bonald, A.Proutiere, G.Regnie, and J.Roberts, "Statistical bandwidth sharing: A study of congestion at flow level," in Proceedings of ACM SIGCOMM, Pitsburgh, PA, 2002.

[6] Kevin Thompson, Gregory J. Miller, and Rick Wilder, "Wide-area internet traffic patterns and characteristics," IEEE Network, vol. 11, no. 6, 1997. 
[7] C.Fraleigh, S.Moon, C.Diot, B.Lyles, and F.Tobagi, "Packet-level traffic measurements from a Tier-1 IP backbone," Tech. Rep. TR01-ATL-110101, Sprint ATL Technical Report, Nov. 2001.

[8] Vern Paxson and Mark Allman, "Computing TCP's retransmission timer," RFC2988, Nov. 2000.

[9] Wael Noureddine and Fouad Tobagi, "Improving the performance of interactive TCP applications using service differentiation," in Proceedings of IEEE INFOCOM, New York, NY, 2002.

[10] Lian Guo and Ibrahim Matta, "The war between mice and elephants," in Proceedings of the 9th IEEE International Conference on Network Protocols ICNP'01, 2001.

[11] Liang Guo and Ibrahim Matta, "Differentiated control of web traffic: A numerical analysis," in Proceedings of SPIE ITCOM'2002: Scalability and Traffic Control in IP Networks, Boston, MA, 2002.

[12] Liang Guo and Ibrahim Matta, "Scheduling flows with unknown sizes: Approximate analysis," Tech. Rep. BU-CS-2002-009, Boston University, Mar. 2002.

[13] I. Rai, G. Urvoy-Keller, and E. Biersack, "Size-based scheduling with differentiated services to improve response time of highly varying flows," in Proceedings of the 15th ITC Specialist Seminar, Internet Traffic Engineering and Traffic Management, Wurzburg, Germany, 2002.

[14] I. Rai, G. Urvoy-Keller, and E. Biersack, "Analysis of LAS scheduling for job size distributions with high variance," in Proceedings of ACM Sigmetrics 2003, International Conference on Measurement and Modeling of Computer Systems, 2003.

[15] L. Massoulié and J. Roberts, "Bandwidth sharing and admission control for elastic traffic," Telecommunication Systems, , no. 15, pp. 185-201, 2000.

[16] E.G. Coffman, "Stochastic models of multiple and time-shared computer operation," Tech. Rep. 66-38, Department of Engineering, University of California at Los Angeles, June 1966.

[17] E.G. Coffman and L. Kleinrock, "Feedback queueing models for time-shared systems," Journal of the Association for Computing Machinery, , no. 15, pp. 549-576, 1968.

[18] L.E. Schrage, "The queue M/G/1 with feedback to lower priority queues," Management Science, , no. 13, pp. 466-471, 1967.

[19] M. Harchol-Balter, K. Sigman, and A. Wierman, "Asymptotic convergence of scheduling policies with respect to slowdown," Performance Evaluation, , no. 49, pp. 241-256, 2002.

[20] S.Bellovin, "Defending against sequence number attacks," RFC 1948, May 1996. 
[21] Hari Balakrishnan, Venkata Padmanabhan, Srinivasan Seshan, Mark Stemm, and Randy Katz, "TCP behavior of a busy web server: analysis and improvements," in Proceedings of IEEE INFOCOM, San Francisco, CA, 1998.

[22] "Network simulator, ver.2, (ns-2)," available at http://www-mash.cs.berkeley.edu/ns.

\section{Appendix. Proof of Theorem 1}

First we note that the solution of the integral equation (7) is equivalent to the solution

$$
\begin{aligned}
\delta \alpha^{\prime}(x)= & \lambda \bar{n} \int_{0}^{\infty} \delta \alpha^{\prime}(y) B(x+y) d y \\
& +\lambda \bar{n} \int_{0}^{x} \delta \alpha^{\prime}(y) B(x-y) d y \\
& +b B(x),
\end{aligned}
$$

where

$$
\delta \alpha^{\prime}(x):=\alpha^{\prime}(x)-\frac{1-\rho_{t h}}{1-\rho} .
$$

The above transformation removes the constant component of the solution. Next, we consider the fixed point iterations

$$
\begin{aligned}
\delta \alpha_{k+1}^{\prime}(x)= & \lambda \bar{n} \int_{0}^{\infty} \delta \alpha_{k}^{\prime}(y) B(x+y) d y \\
& +\lambda \bar{n} \int_{0}^{x} \delta \alpha_{k}^{\prime}(y) B(x-y) d y \\
& +b B(x), \quad k=0,1, \ldots
\end{aligned}
$$

on the complete functional space of continuous bounded positive functions $\mathcal{C}[0, \infty)$ with the supremum metric. Let $M=\left\|\delta \alpha^{\prime}\right\|=\sup _{x}\left|\delta \alpha^{\prime}(x)\right|<\infty$.

If we show that the linear integral operator

$$
\begin{aligned}
\mathcal{A}[\beta(x)]= & \lambda \bar{n} \int_{0}^{\infty} \beta(y) B(x+y) d y \\
& +\lambda \bar{n} \int_{0}^{x} \beta(y) B(x-y) d y+b B(x)
\end{aligned}
$$

is a contraction, then the integral equation (7) has a unique solution in $\mathcal{C}[0, \infty)$. Clearly the operator $\mathcal{A}[\beta(x)]$ maps the space $\mathcal{C}[0, \infty)$ into itself.

Let us denote as $d$ the distance in the metric space $\mathcal{C}[0, \infty)$, that is $d\left(\delta \alpha_{1}^{\prime}, \delta \alpha_{2}^{\prime}\right)=$ $\sup _{x}\left\{\left|\delta \alpha_{1}^{\prime}-\delta \alpha_{2}^{\prime}\right|\right\}$. Let us now show that the linear operator $\mathcal{A}[\beta(x)]$ is indeed a contraction 
mapping on $\mathcal{C}[0, \infty)$.

$$
\begin{aligned}
d\left(\mathcal{A}\left[\delta \alpha_{1}^{\prime}\right], \mathcal{A}\left[\delta \alpha_{2}^{\prime}\right]\right)= & \sup _{x}\left\{\left|\mathcal{A}\left[\delta \alpha_{1}^{\prime}\right]-\mathcal{A}\left[\delta \alpha_{2}^{\prime}\right]\right|\right\} \\
\leq & \lambda \bar{n} \sup _{x}\left\{\mathcal{A}\left[\mid \delta \alpha_{1}^{\prime}\right]-\mathcal{A}\left[\delta \alpha_{2}^{\prime}\right] \mid\right\} \\
& \sup _{x}\left(\int_{0}^{\infty} B(x+y) d y+\right. \\
& \left.\int_{0}^{x} B(x-y) d y\right) \\
= & \lambda \bar{n} d\left(\delta \alpha_{1}^{\prime}, \delta \alpha_{2}^{\prime}\right) \frac{X-X_{t h}}{1-\rho_{t h}} \\
= & d\left(\delta \alpha_{1}^{\prime}, \delta \alpha_{2}^{\prime}\right) \frac{\rho-\rho_{t h}}{1-\rho_{t h}} .
\end{aligned}
$$

Thus, the mapping is a contraction if $\rho<1$.

We now show that $\lim _{x \rightarrow \infty} \delta \alpha^{\prime}(x)=0$. We note that $\lim _{x \rightarrow \infty} B(x)=0$. Clearly the first integral tends to zero as $x \rightarrow \infty$. Then we have:

$$
\begin{aligned}
& \limsup _{x \rightarrow \infty} \delta \alpha^{\prime}(x)=\frac{\lambda \bar{n}}{1-F(t h)} \times \\
& \times \limsup _{x \rightarrow \infty} \int_{0}^{x} \delta \alpha^{\prime}(y)(1-F(x-y+t h)) d y
\end{aligned}
$$

We note that $1-F(x-y+t h)$ is an increasing function on $y$ and $\lim _{y \rightarrow \infty}(1-F(x-$ $y+t h))=1$. We choose $x^{*}<x$ to be large enough so $\forall x>x^{*}, \delta \alpha^{\prime}(x)<\lim \sup _{z \rightarrow \infty} \delta \alpha^{\prime}(z)$. Let us denote $1-F\left(x-x^{*}+t h\right)=\varepsilon$, then

$$
\begin{aligned}
\delta \alpha^{\prime}(x) \leq & \frac{\lambda \bar{n}}{1-F(t h)}\left(x^{*} \varepsilon M+\limsup _{z \rightarrow \infty} \delta \alpha^{\prime}(z)\right) \\
& \int_{x^{*}}^{x}(1-F(x-y+t h)) d y .
\end{aligned}
$$

Now we take the $\lim \sup _{x \rightarrow \infty}$ of the above expression. We note that the first component tends to 0 since $\lim \sup _{x \rightarrow \infty} \varepsilon=\lim _{x \rightarrow \infty}\left(1-F\left(x-x^{*}+t h\right)\right)=0$. Integrating by parts it is easy to see that $\int_{0}^{x}(1-F(y)) d y=\bar{X}_{x}$. Then we obtain

$$
\begin{aligned}
\limsup _{x \rightarrow \infty} \delta \alpha^{\prime}(x) \leq & \frac{\lambda \bar{n}}{1-F(t h)} \limsup _{z \rightarrow \infty} \delta \alpha^{\prime}(z) \\
& \limsup _{x \rightarrow \infty} \int_{x^{*}}^{x}(1-F(x-y+t h)) d y \\
= & \frac{\lambda \bar{n}}{1-F(t h)} \limsup _{z \rightarrow \infty} \delta \alpha^{\prime}(z)
\end{aligned}
$$




$$
\begin{aligned}
& \limsup _{x \rightarrow \infty} \frac{X_{x-x^{*}-t h}-\overline{X_{t}} h}{1-F(t h)} \\
= & \frac{\rho-\rho_{t h}}{1-\rho_{t h}} \limsup _{x \rightarrow \infty} \delta \alpha^{\prime}(x) .
\end{aligned}
$$

Since $\left(\rho-\rho_{t h}\right) /\left(1-\rho_{t h}\right)<1$ if $\rho<1$, the equality holds only if $\lim \sup _{x \rightarrow \infty} \delta \alpha^{\prime}(x)=0$. Consequently, the limit exists and is equal to zero. Furthermore, since $\delta \alpha^{\prime}(x) \in \mathcal{C}[0, \infty)$ and $\lim _{x \rightarrow \infty} \delta \alpha^{\prime}(x)=0$, using equation (6), we conclude that $\lim _{x} \bar{S}^{P S+P S}(x)=1 /(1-\rho)$

To have an asymptote, the integral of $\delta \alpha^{\prime}(x)$ must be bounded.

$$
\begin{aligned}
& \int_{0}^{\infty} \delta \alpha^{\prime}(x) d x= \\
& =\lambda \bar{n} \int_{0}^{\infty} \int_{0}^{\infty} \delta \alpha^{\prime}(y) B(x+y) d y d x \\
& +\lambda \bar{n} \int_{0}^{\infty} \int_{0}^{x} \delta \alpha^{\prime}(y) B(x-y) d y d x \\
& +b \int_{0}^{\infty} B(x) d x \\
& =\lambda \bar{n} \int_{0}^{\infty} \delta \alpha^{\prime}(x)\left(\int_{y}^{\infty} B(x) d x+\int_{0}^{\infty} B(x) d x\right) d y \\
& +b \int_{0}^{\infty} B(x) d x \\
& \leq \lambda \bar{n} 2 \int_{0}^{\infty} B(x) d x \int_{0}^{\infty} \delta \alpha^{\prime}(y) d y+b \int_{0}^{\infty} B(x) d x \\
& =\lambda \bar{n} 2 \frac{\bar{X}-\bar{X}_{t h}}{1-F(t h)} \int_{0}^{\infty} \delta \alpha^{\prime}(y) d y+b \frac{\bar{X}^{-}-\bar{X}_{t h}}{1-F(t h)} \\
& =2 \frac{\rho-\rho_{t h}}{1-\rho_{t h}} \int_{0}^{\infty} \delta \alpha^{\prime}(y) d y+b \frac{\bar{X}-\bar{X}_{t h}}{1-F(t h)}
\end{aligned}
$$

If $2 \rho-\rho_{t h}<1$ then $2\left(\rho-\rho_{t h}\right) /\left(1-\rho_{t h}\right)<1$ and the integral of $\delta \alpha^{\prime}(x)$ is bounded by

$$
\int_{0}^{\infty} \delta \alpha^{\prime}(y) d y \leq \frac{b\left(\bar{X}-\bar{X}_{t h}\right)\left(1-\rho_{t h}\right)}{(1-F(t h))\left(1+\rho_{t h}-2 \rho\right)}
$$

Using equation (6) we conclude that $\bar{T}^{P S+P S}(x)$ has an asymptote with the slope $1 /(1-\rho)$, if $2 \rho-\rho_{t h}<1$. This completes the proof. 


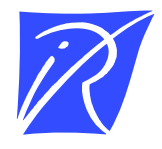

\section{Unité de recherche INRIA Sophia Antipolis 2004, route des Lucioles - BP 93 - 06902 Sophia Antipolis Cedex (France)}

Unité de recherche INRIA Futurs : Parc Club Orsay Université - ZAC des Vignes 4, rue Jacques Monod - 91893 ORSAY Cedex (France)

Unité de recherche INRIA Lorraine : LORIA, Technopôle de Nancy-Brabois - Campus scientifique 615, rue du Jardin Botanique - BP 101 - 54602 Villers-lès-Nancy Cedex (France)

Unité de recherche INRIA Rennes : IRISA, Campus universitaire de Beaulieu - 35042 Rennes Cedex (France)

Unité de recherche INRIA Rhône-Alpes : 655, avenue de l'Europe - 38334 Montbonnot Saint-Ismier (France)

Unité de recherche INRIA Rocquencourt : Domaine de Voluceau - Rocquencourt - BP 105 - 78153 Le Chesnay Cedex (France)

INRIA - Domaine de Voluceau - Rocquencourt, BP 105 - 78153 Le Chesnay Cedex (France)

http://www.inria.fr

ISSN 0249-6399 